\title{
Eigen Trust Algorithm for Resource Selection in Grid Computing
}

\author{
Vivekananth.P \\ Lecturer \\ BOTHO COLLEGE \\ Gaborone, Botswana
}

\begin{abstract}
Grid Computing is an enhanced form of distributed computing where the resources are shared among the heterogeneous resources. The primary goal Grid Computing is to reduce the overall elapsed processing time while leveraging existing hardware. A Grid Computing system is a geographically distributed system with different domains, sharing resources. Security plays very important role in any Internet system, specifically in Grid Computing system. Since all resources are shared in a Grid computing system, trust relationship is very crucial. Selecting appropriate resources within such a distributed environment is a tedious task. This paper proposes an approach for resource selection based on reputation.
\end{abstract}

Keywords: Reputation, trust, Eigen trust

\section{INTRODUCTION}

Grid Computing is an enhanced form of distributed computing. The primary goal Grid Computing is to reduce the overall elapsed processing time while leveraging existing hardware. There are two types of Grid namely, "Utility" grid and a "Compute" grid. The concept of the "Utility" grid is quite simple. On one side we have a pool of users who need to do some processing. On the other side we have some available computing machines or CPUs, and dynamically a user or process gets assigned to an available machine. This is somewhat like the use of a Mainframe computer. We submit a job, a virtual machine processes it and we get back the results, without knowing how or where exactly it was processed.

The difference in the Grid environment, of course, is that the CPUs can be located physically on different machines. The idea behind the "Compute" grid is that we are able to break up a task into sub-tasks, which we then can submit to available

machines, which then means that theses sub-tasks can then run in parallel or at the same time.

A Grid Computing system is a geographically distributed system with different domains, sharing resources. Security plays very important role in any Internet system, specifically in Grid Computing system. Since all resources are shared in a Grid computing system, trust relationship is very crucial. Selecting appropriate resources within such a distributed environment is a tedious task. A Reputation management Service is implemented in this project, which evaluates the selection of a resource based on Reputation. Grid computing [2] initially focused on largescale resource sharing, innovative applications, and achievement of high-performance. Today, the Grid approach [3] suggests the development of a distributed service environment that integrates a wide variety of resources with various qualities of service capabilities to support scientific and business problem solving environments. However, optimal utilization of these distributed services and resources often require the Grid user to make a prudent decision regarding the capacity of these remote resources. Users are faced with questions such as: which resources are available remotely, what capabilities do these resources have, am I authorized to use these resources, and so on. A reputation based trust model is developed which selects the appropriate resource based on reputation. Reputation is computed using a trust rating provided by users of services through a feedback mechanism. Reputation-based service and product selection has proved to be a great asset for online site eBay [7]. The rest of the chapters are organized as follows. Section 2 discusses about literature survey, section 3 explains about the model which uses eigen trust for resource selection and section 4 is the conclusion.

\section{LITERATURE SURVEY}

Beulah Kurian Alunkal and Ivana Veljkovic, et al in their paper [1] explain how to select the best resource based on Reputation. S.D Kamvar and M.T Schlosser et al [4] in their paper explain how the eigen trust algorithm ensures security in a peer to peer networks. The paper by Hotel Fort Garry, Winnnpeg [5] explains how the direct trust can be calculated. G. Von Laszewski and S. Fitzgerald et al [6] design a Globus Monitoring and Directory Service (MDS) that provides uniform, efficient and scalable access to dynamic, distributed and diverse information about the structure and state of the resources. However the information returned by this service is inaccurate or outdated and does not integrate a resource selection service. The paper "Trusted grid computing with security assurance with resource optimization " by Shanshan song and Kai Hwang suggests a model which aims at securing grid resources with optimized resources subject to budget constraints. It works well with increasing number of divisible jobs and sustain efficiency even when new sites are added. Fuzzy logic is used to build the trust model. Based on previous job execution the trust worthiness is measured[7]. 


\subsection{Applications of Reputation An example: ebay}

If people want to sell items on e-bay, they list and describe their items generally with digital photos-at http://www.ebay.com. They set a minimum bid, decide on the length of the auction $(3,5,7$ or 10 days), and disclose acceptable forms of payment. To place a bid, a bidder enters an e-bay username and password along with a maximum bid. The ebay system then compares that bid with any other bids on the same item naming a high bid above the next highest maximum bid. When time expires for the auction, both high bidder and seller are notified by e-mail of the auction's end and of the winning bid. The high bidder and seller are then supposed to make contact with each other via e-mail within three days, at which time they exchange addresses. Both buyers and sellers must trust in order for transactions to take place.

\section{eBay solution:}

Trust in Community

\section{Motto of eBay:}

We believe people have something to contribute.

We believe people are basically good.

If you are a buyer, check your seller's feedback profile before you enter into a transaction to learn about the other person's reputation with previous buyers. If you are seller do the same with your buyers.

\section{THE PROPOSED MODEL}

This model facilitates the best resource selection based on reputation. The terminology used in this model is defined as follows.

\section{Trust}

Trust is a mechanism for reducing risk in unknown situations and expectation about behavior.

\section{Reputation}

Reputation refers to the value given to the entity (it may be a resource, service, user) based on the trust exhibited by it in the past. It reflects the perception that one has of another's intentions and norms.

\section{Reputation Service}

A reputation service is defined as a secure informative service responsible for maintaining a dynamic trust and reputation metric for its community.

\section{Providers}

A provider in the Grid environment is the one, which provides the resource.

\section{Consumers}

A consumer in the Grid environment is the one, which consumes the resource. A resource can be a consumer as well as a provider.

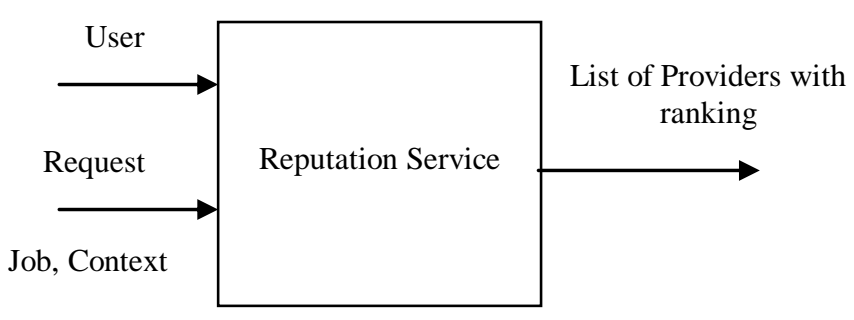

Figure 1 Reputation Model

\subsection{Eigen Trust Algorithm}

The Eigen Trust algorithm involves the following steps. The following assumptions are made. If the resource requested is the printer, the providers are taken from 31 to 40 .If the resource requested is computing the providers are considered from 41 to 50. If the resource requested is data then the providers are 51 to 60.

The steps in Eigen Trust algorithm are as follows:

- The feedback values are collected for the respective providers from each consumer table.

- The sum of transactions of each provider is calculated from each consumer table. If the sum of the transaction of the provider is less than zero then the sum of the particular provider is omitted, and the sum of all the transaction of the other providers are calculated.

- The sum of the values is normalized.

- After normalization the values are represented in the form of a matrix. (a $n$ by $n$ matrix.)

- The transpose of a matrix is taken, and the power of the matrix is taken and it is found that they converge to a single value.

- The reputation of the resource is given by the formula

$\frac{\alpha \text { (Direct trust) }+\beta \text { (global reputation) }}{\alpha+\beta}$

where $\alpha$ and $\beta$ are weight ages given to direct trust and global reputation respectively. The weight age given for $\alpha$ is 0.8 and weight age given for $\beta$ is 0.2 .

- The top four reputed resources are ranked in the order of trust.

\section{EXPERIMENTS AND RESULTS}

The consumers simulate the request for a resource randomly. The consumers can request for the resource printer, computing and data. Any consumer from 11 to 30 can request for the resource printer. Any consumer from 1 to 10 and from 21 to 30 can request for the resource computing. Any consumer from 1 to 20 can request for the resource data. The providers 31 to 40 provide the resource printer. The providers 41 to 50 provide the resource computing. The providers 51 to 60 provide the resource data. 
If a consumer requests for the resource printer then the sum of the feedback values of the providers 31 to 40 is taken from the consumer tables 1 to 10 . After calculating the sum, the values are normalized. If the sum of the feedback value is negative, the value is normalized to zero. The normalized values are represented in the form of 30 by 30 matrix. The 30 by 30 matrix denotes 30 consumers and 30 providers. Only those consumers that provide the resource printer have the values represented in the form of matrix. The values are zero in other rows and columns. The transpose of 30 by 30 matrix is taken, and it is raised to the power10. It is found that the matrix when raised to the power 10 converges to a single value. The consumers 1 to 10 calculate the reputation of the resource for the providers 31 to 40.The reputation of the resource printer given by consumer1 for provider 31 is calculated using the formula.

\section{$0.8 *$ direct trust $+0.2 *$ global reputation}

Direct trust- the value given by consumer1 for provider31 that is represented in the form of matrix.

Global reputation-The value given by consumer 1 for provider31 after the matrix is raised to the power 10.

The consumers 1 to 10 calculate the reputation of the resource printer for the providers 31 to 40 .The top four reputed resources are ranked in the order of trust. The Reputation of the resource printer is depicted in figure 4.1 .

If a consumer requests for the resource data then the sum of the feedback values of the providers 51 to 60 is taken from the consumer tables 21 to 30 . After calculating the sum, the values are normalized. If the sum of the feedback value is negative, the value is normalized to zero. The normalized values are represented in the form of 30 by 30 matrix. The 30 by 30 matrix denotes 30 consumers and 30 providers. Only those consumers that provide the resource data have the values represented in the form of matrix. The values are zero in other rows and columns. The transpose of 30 by 30 matrix is taken, and it is raised to the power10. It is found that the matrix when raised to the power 10 converges to a single value. The consumers 21 to 30 calculate the reputation of the resource for the providers 51 to 60.The reputation of the resource data given by consumer 21 for provider 51 is calculated using the figure (4.1)

The consumers 21 to 30 calculate the reputation of the resource computing for the providers 51 to 60 .The top four reputed resources are ranked in the order of trust. The reputation of the resource data is depicted in figure 4.2 .

If a consumer requests for the resource computing then the sum of the feedback values of the providers 41 to 50 is taken from the consumer tables 11 to 20 . After calculating the sum, the values are normalized. If the sum of the feedback value is negative, the value is normalized to zero. The normalized values are represented in the form of 30 by 30 matrix. The 30 by 30 matrix denotes 30 consumers and 30 providers. Only those consumers that provide the resource computing have the values represented in the form of matrix. The values are zero in other rows and columns. The transpose of 30 by 30 matrix is taken, and it is raised to the power10. It is found that the matrix when raised to the power 10 converges to a single value. The consumers 11 to 20 calculate the reputation of the resource for the providers 41 to 50.The reputation of the resource computing given by consumer 11 for provider 41 is calculated using the figure 4.1

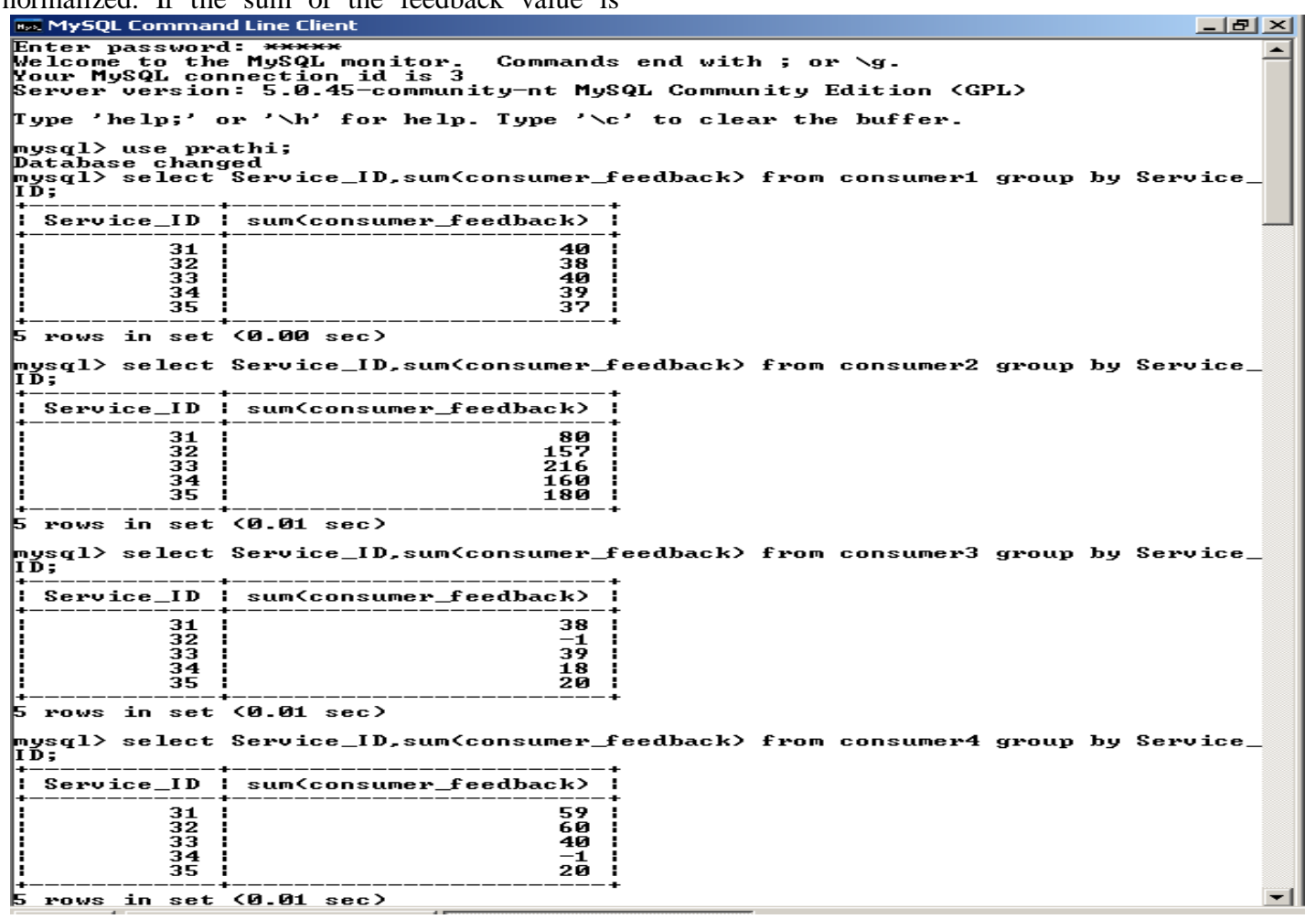

Figure 4.1 Feedback table 
Figure 4.2 depicts cumulative sum of feedbacks of the providers $31,32,33,34,35$ given by consumer 1 ,consumer2, consumer3, consumer4, and consumer 5 for the resource printer. Figure 4.3 Consumer feedbacks summed for the resource computing. The reputation of the resource computing is depicted in fig 4.2 to fig.4. 3.

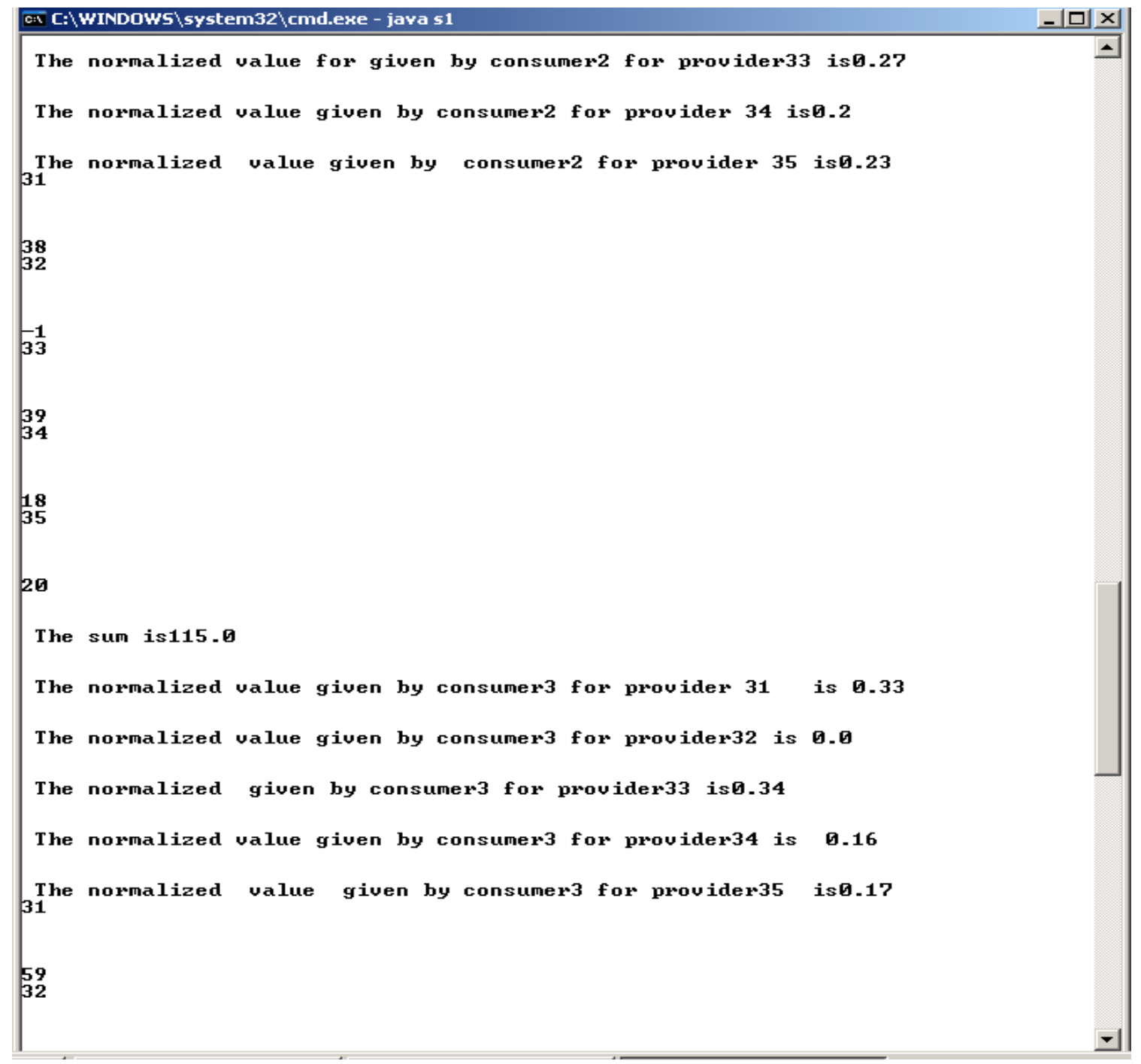

Figure 4.2 Snapshot for Consumer17 requisition for resource printer. 


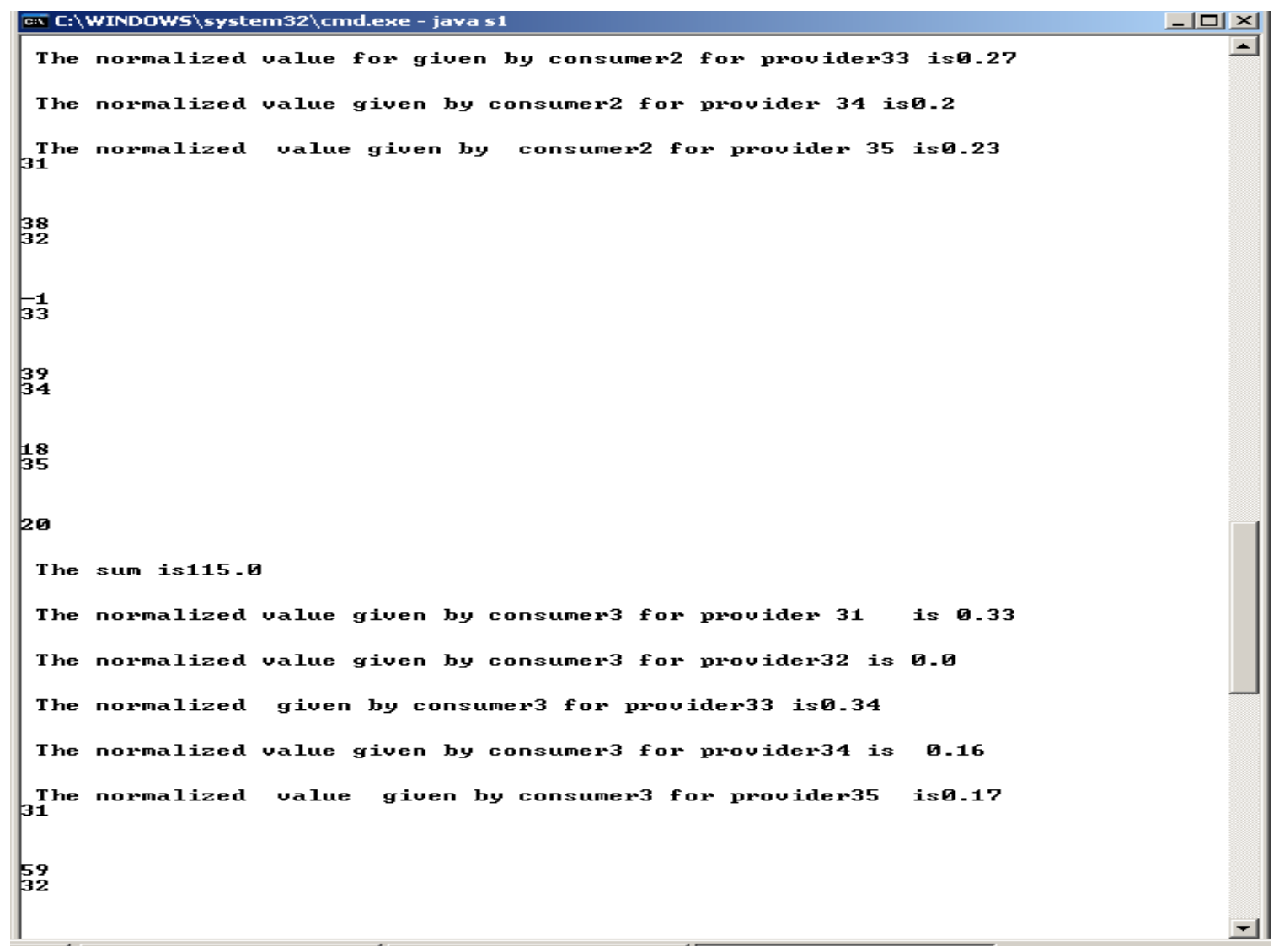

Figure 4.3 consumer feed backs

\section{CONCLUSION}

Grid resource selection is becoming more and more important topic, a number of problems still remain to be tackled by the current Grid solutions. Group-oriented security and distributed system behavior conformance are identified among the essential requirements for Grid resource selection. This paper proposed a reputation based trust model by using eigen trust algorithm for resource selection.

\section{REFERENCES}

[1] Beulah kurian, Gregor von lazuli ,(2003) 'Reputation based grid resource selection' in the proceedings of the workshop on adoptive resource selection ,pp 28-36.

[2] Foster and C.Kesselman, Eds. (1998) "The Grid: Blueprint for a New Computing Infrastructure". Morgan Kaufmann Publishers.

[3] G.Von Laszewski,G. Pieper , and P.Wagstrom (2003), "Gestalt of the Grid", in Performance Evaluation and Characterization of Parallel and Distributed Computing Tools, ser. Series on Parallel and Distributed Computing.

[4] S.D Kamvar, M.T Schlosser, and H. Garcia-Molina (2003), "The Eigen trust algorithm for reputation management in p2p networks," ACM Press.
[5]. Hotel Fort Gary,Winnipeg, and Manitoba (2002),Evolving and Managing Trust in Grid Computing Systems,IEEE computer Society Press.

[6]G.VonLaszewski,S.Fitzgerald ,I.Foster,C.Kesselman,W.Smith and S.Tuecke (1997),"A Directory Service for Configuring High-Performance Distributed Computations", in Proceedings of the 6th IEEE Symposium on HighPerformance Distributed Computing,365-375.

[7] Shanshan song and Kai Kwang "Trusted Grid Computing with security assurance and resource optimization " in proceedings of the 17 th international conference on parallel and distributed computing systems " September-2004.

8 Srivaramangai P Renagaramanujam Srinivasan Reputation Based Two Way Trust Model for Reliable Transactions in Grid Computing, Published in Volume 7, Issue 3, No 5, pp 33-39, May 2010. 